\title{
Measurement of the Solar Magnetic Field effect on cosmic rays using the Sun shadow observed by the ARGO-YBJ experiment
}

\author{
Songzhan Chen*i \\ Key Laboratory of Particle Astrophysics, Institute of High Energy Physics, CAS, Beijing 100049, \\ China. \\ E-mail: Chenszeihep.ac.cn

\section{Yuncheng Nan} \\ Key Laboratory of Particle Astrophysics, Institute of High Energy Physics, CAS, Beijing 100049, \\ China. \\ University of Chinese Academy of sciences, Beijing 100049, China.
}

\section{On behalf of the ARGO-YBJ Collaboration}

\begin{abstract}
The cosmic rays originating outside the solar system are blocked by the Sun and form a shadow in the sky map, whose shape is affected by the magnetic field between the Sun and the Earth. Therefore the Sun shadow can be used as a probe of the magnetic field and its intensity variations. In this work, we report on the observation of the rigidity dependent variation of the Sun shadow signal in the rigidity range 0.4-200 TV, using the ARGO-YBJ data collected in the years from 2008 to 2012, when the solar activity varied from the minimum to the maximum. The Moon shadow is also investigated and used to remove the geomagnetic effect on the Sun shadow. According to our measurement, the solar magnetic field significantly affects the flux of cosmic rays with energy lower than $100 \mathrm{TV}$, that appear to be strongly correlated with the solar activity, while at higher rigidity the solar influence is less evident. We also discuss the possibility to study the different magnetic field components around the Sun.
\end{abstract}

35th International Cosmic Ray Conference - ICRC2017

10-20 July, 2017

Bexco, Busan, Korea

\footnotetext{
*Speaker.

${ }^{\dagger}$ This work is supported in China by NSFC No.11575203
} 


\section{Introduction}

Cosmic rays are high energy particles from outside the solar system, which are mainly composed of positively charged nuclei. Due to the modulation of interstellar magnetic field, cosmic rays usually arrive at the Earth nearly isotropically. The anisotropy in different directions is less than $0.2 \%$ at energies from sub TeV to PeV. The Moon and Sun are the two largest apparent objects in the sky with optical diameter of about $0.5^{\circ}$ viewed from the Earth. In 1957, Clark suggested that the Sun and Moon must cast shadows in the cosmic ray intensity and observation of their shadows might provide information about the magnetic field of these bodies [U].

Since the first measurement of the combined shadow of Sun and Moon obtained by the Cygnus group in 1991 at $4.9 \sigma$ level [व], the Moon shadow and Sun shadow have been widely detected by different experiments. Now, the Moon shadow has been taken as an important tool to check the performance of an extensive air shower (EAS) array, e.g., angular resolution, absolute pointing accuracy. The charged cosmic rays are deflected by the geomagnetic field (GMF) when they pass close to the Moon towards the Earth, thus the Moon shadow is displaced with respect to the Moon position. Knowing the GMF configuration with sufficient precision, the displacement of Moon shadow has been well studied and has also been used to calibrate the absolute rigidity scale of cosmic rays by EAS arrays [B] .

The Sun shadow is affected by all the magnetic fields between the Sun and the Earth, including the solar magnetic field (SMF) near the Sun, the interplanetary magnetic field (IMF), and also the GMF. Therefore, the Sun shadow can be used as a probe of these magnetic fields and their intensity variations. The effect of GMF on the Sun shadow is stable and very similar to that on the Moon shadow. The IMF is the component of the SMF which is dragged out from the solar corona by the solar wind flow to fill the solar System. The IMF is usually monitored by orbiting detectors near the Earth. The IMF is strongly modulated by solar activities, while the large-scale structure of the IMF is fairly simple and stable in the quiet phase. In 2011, the ARGO-YBJ collaboration has quantitatively measured the intensity of IMF for the first time using the displacement of Sun shadow in the north-south direction during the solar quiet phase [四]. The SMF is strong and complex. It is connected with the 11-year cycle of solar activity and has not been fully understood yet. In 2013, the Tibet AS $\gamma$ collaboration has tested the coronal magnetic field models, i.e., the potential field source surface (PFSS) model and the current sheet source surface (CSSS) model, by using the deficit ratio of Sun shadow at $10 \mathrm{TeV}$ [可].

According to the AS $\gamma$ measurement at $10 \mathrm{TeV}$, the Sun shadow is deeply modulated by the solar activity. The shadow is significant during the solar quiet phase, while it almost disappears during the maximum of activity [5]. The ARGO-YBJ observation around $5 \mathrm{TeV}$ also shows a similar tendency [目]. In the previous analysis, only one energy point has been considered. Actually, the effect of magnetic field on charged particles depends on the particle rigidity, and it is expected to decrease with increasing rigidity. It would be important to verify this dependence, which has not yet been observed by current EAS arrays. On the other hand, the effects of SMF and IMF are complex and strictly correlated, especially during the solar active phase. In such a situation, an observation of the rigidity dependent variation of the Sun shadow will provide more information on the magnetic field and its intensity variations. The aim of this work, is to measure the rigidity dependent variation of the Sun shadow in the range 0.4-200 TV using the ARGO-YBJ data from 
2008 to 2012, when the solar activity varied from the minimum to the maximum. To evaluate the effect of GMF on the Sun shadow, the Moon shadow is also investigated using the same data sample.

\section{The ARGO-YBJ experiment data}

The ARGO-YBJ experiment (Tibet, China at $4300 \mathrm{~m}$ a.s.l.) consists of a single layer of Resistive Plate Chambers (RPCs) with a $74 \times 78 \mathrm{~m}^{2}$ carpet (93\% coverage) in the center and a surrounded partially instrument ( $20 \%$ coverage) extending the whole area to $100 \times 110 \mathrm{~m}^{2}$. More details about the detector can be found in $[\square]$. The trigger rate is $3.5 \mathrm{kHz}$ with a dead time of $4 \%$ and an average duty-cycle higher than $86 \%$. It can detect cosmic rays from about $300 \mathrm{GeV}$ to $\mathrm{PeV}$ energies. With a field of view of $2 \mathrm{sr}$, ARGO-YBJ can instantaneously monitor the whole overhead sky with zenith angle $\theta<50^{\circ}$, making possible to observe the Sun shadow during most of days in the year. Figure 1 shows the ideal daily allowable Sun observation time for ARGO-YBJ during the full ARGOYBJ operation time from 2007 November to 2013 February. The maximum allowable observation time in one day is 7.4 hours. To investigate the yearly variation of the Sun shadow, the data taken from 2008 to 2012 are used in this work. During this period, the allowable observation time for ARGO-YBJ is 8572.9 hours, while the real observation time is 7471.9 hours, as shown in Figure 1.

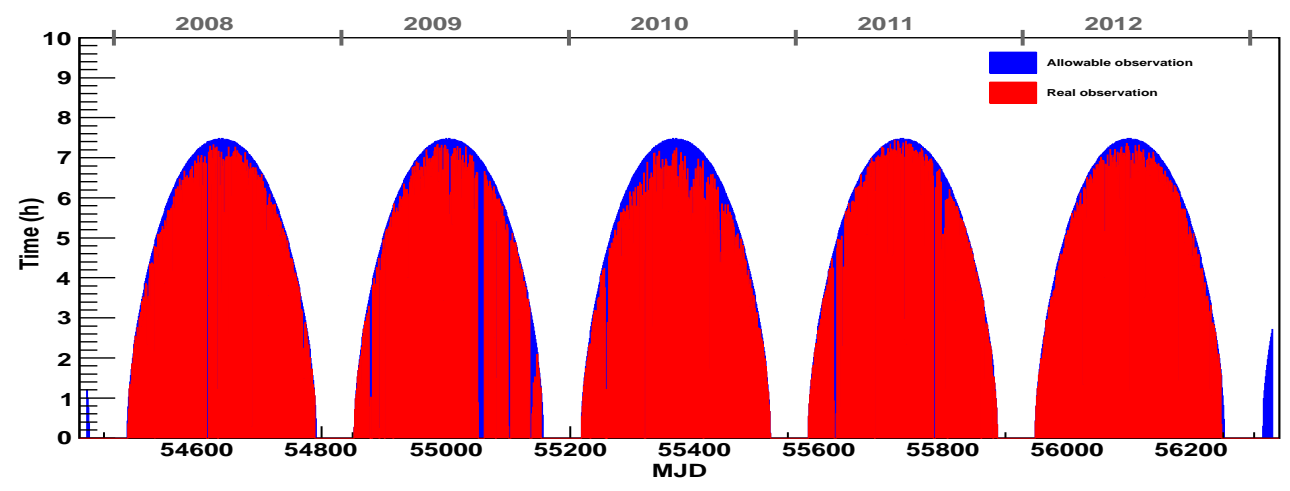

Figure 1: Daily allowable observation time and real observation time of ARGO-YBJ for the Sun. The zenith angle of Sun is required to be less than $50^{\circ}$.

To estimate the performances of ARGO-YBJ detector for cosmic rays from the Sun direction, the Sun is traced during one complete year of observation, i.e., 365 transits. Then a full Monte Carlo simulation process is implemented using CORSIKA 6.502 [] [ ] to describe the EAS development in the atmosphere and the GEANT4-based code G4argo [Q] to describe the detector response. For the primary cosmic rays, the phenomenological composition model derived in [ए0] is adopted here. This model has taken into account the new measurements of CREAM using five groups of nuclei, $\mathrm{H}, \mathrm{He}, \mathrm{CNO}, \mathrm{Mg}-\mathrm{Si}$ and Fe. The energy range is from $10 \mathrm{GeV}$ to $1 \mathrm{PeV}$. The shower core location is sampled inside an area of $1000 \times 1000 \mathrm{~m}^{2}$ around the carpet center.

For the analysis presented in this paper, events are selected according to the following cuts: (1) zenith angle $\theta<50^{\circ}$; (2) the distance between the shower core position and the carpet center 
less than $100 \mathrm{~m}$; (3) the time spread of the shower front in the conical fit defined in Equation (1) of [U] less than $100 \mathrm{~ns}^{2}$. In this work, the simulation sample of $\mathrm{H}$ is used to study the energy reconstruction. The primary energy of a shower is mainly related to the number of secondary particles recorded by detector, and also related to the incident zenith angle and core position. The primary cosmic ray energy is reconstructed using these information. Figure 2 shows the relation between the reconstructed energy $\left(\mathrm{E}_{\text {rec }}\right)$ and the primary true energy $\left(\mathrm{E}_{\text {true }}\right)$ for different groups of nuclei. In this work, the events with reconstructed energy from $0.32 \mathrm{TeV}$ to about $1 \mathrm{PeV}$ are divided into 12 energy bins as shown in Table 1 . For each bin, the distribution of primary rigidity taking into account all the groups is also shown in Figure 2. The angular resolution $\left(\sigma_{\text {res }}\right)$, median rigidity for each energy bin are listed in Table 1.
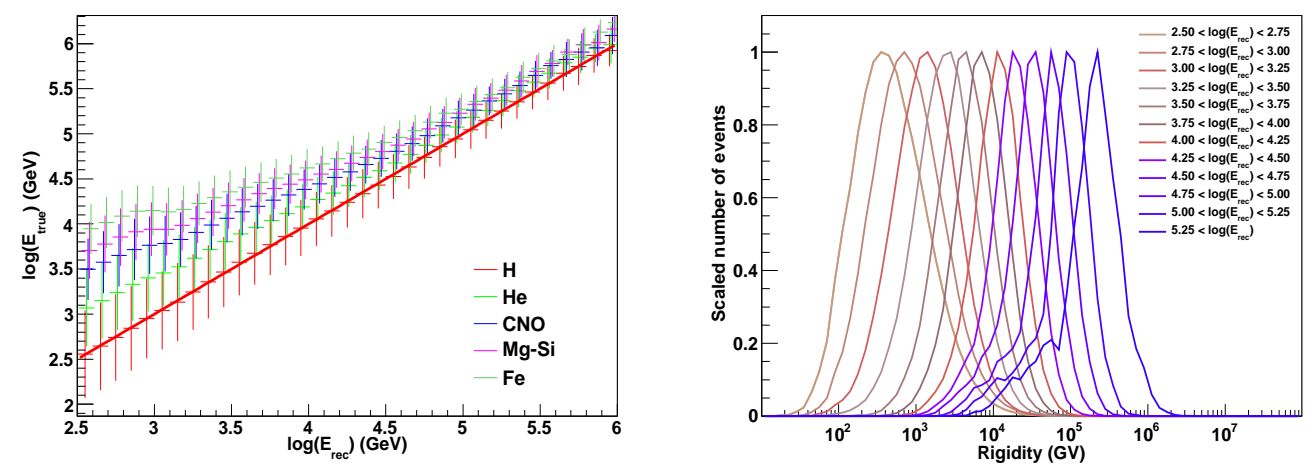

Figure 2: Left:The relation between the reconstructed energy and the primary true energy for different group of nuclei. Right: The distribution of primary rigidity for each energy bin.

Table 1: The angular resolution and median rigidity

\begin{tabular}{cccc}
\hline $\log \left(\mathrm{E}_{\text {rec }}\right)(\mathrm{GeV})$ & $\sigma_{\text {res }}$ & Median Rigidity $(\mathrm{TV})$ & Median Rigidity for Moon shadow(TV) \\
\hline $2.50-2.75$ & $2.36^{\circ}$ & 0.43 & 0.60 \\
$2.75-3.00$ & $1.66^{\circ}$ & 0.75 & 0.97 \\
$3.00-3.25$ & $1.42^{\circ}$ & 1.33 & 1.50 \\
$3.25-3.50$ & $1.10^{\circ}$ & 2.37 & 2.48 \\
$3.50-3.75$ & $0.78^{\circ}$ & 4.12 & 4.12 \\
$3.75-4.00$ & $0.59^{\circ}$ & 6.84 & 6.84 \\
$4.00-4.25$ & $0.48^{\circ}$ & 11.35 & 11.35 \\
$4.25-4.50$ & $0.41^{\circ}$ & 18.41 & 18.84 \\
$4.50-4.75$ & $0.35^{\circ}$ & 31.26 & 32.73 \\
$4.75-5.00$ & $0.31^{\circ}$ & 54.33 & 58.21 \\
$5.00-5.25$ & $0.31^{\circ}$ & 92.26 & 98.86 \\
$>5.25$ & $0.33^{\circ}$ & 197.24 & 201.84 \\
\hline
\end{tabular}

\section{Analysis method and result}

In this analysis, the $40^{\circ} \times 40^{\circ}$ sky region in celestial coordinates center on the Sun (Moon) is 
divided into a grid of $0.1^{\circ} \times 0.1^{\circ}$ pixels and filled with detected events. The number of events in each pixel is denoted as $n$. The "direct integral method" [ए2] is adopted to estimate the number of background events in each pixel (denoted as $b$ ). The integral time is set to be 2 hours in this work. The total number of expected deficit events $S_{\text {ideal }}$ can be evaluated by the number of background events expected in a solid angle corresponding to the Sun (Moon) disk. The deficit events, due to the detector angular resolution (known) and the magnetic field effects (unknown), are spread and shifted with respect to the nominal Sun (Moon) position. The central position of the shadow, and the total number $S$ of detected deficit events are found by minimizing the chi-square quantity within a region of interest $(\mathrm{ROI})$ :

$$
\chi^{2}=\sum_{i}^{R O I}\left[\frac{s_{i}+\left(n_{i}-b_{i}\right)}{\sqrt{n_{i}+\alpha b_{i}}}\right]^{2}
$$

where ROI is the central $20^{\circ} \times 20^{\circ}$ region, $s_{i}$ is the expected number of deficit events in $i$ th pixel, $\alpha$ is the ratio of signal and background exposures, $\sigma_{i}=\sqrt{n_{i}+\alpha b_{i}}$ is the uncertainty of $\left(n_{i}-b_{i}\right)$ [प13]. The distribution of $s_{i}$ follows the point spread function of angular resolution listed in Table 1. 2-D Gaussian functions are assumed here. The MINUIT package [14]] is used to minimize the chi-square quantity to fit the parameters including the position of shadow, total number of deficit event (denoted as $S$ ), and corresponding errors.
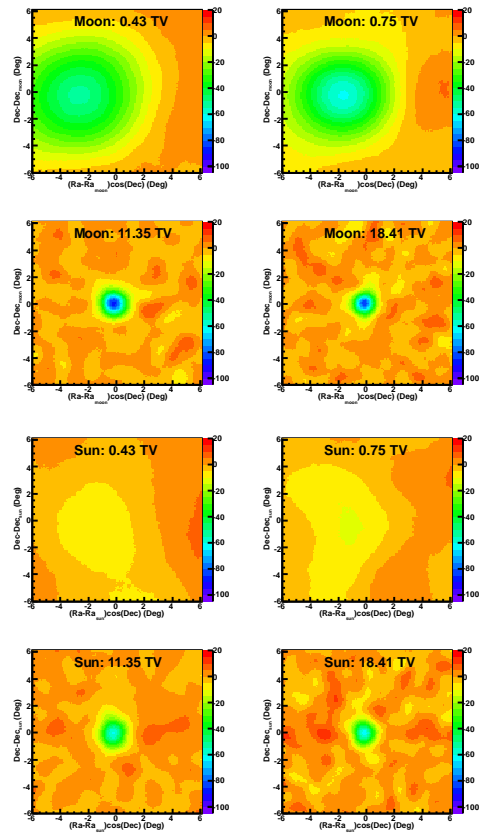
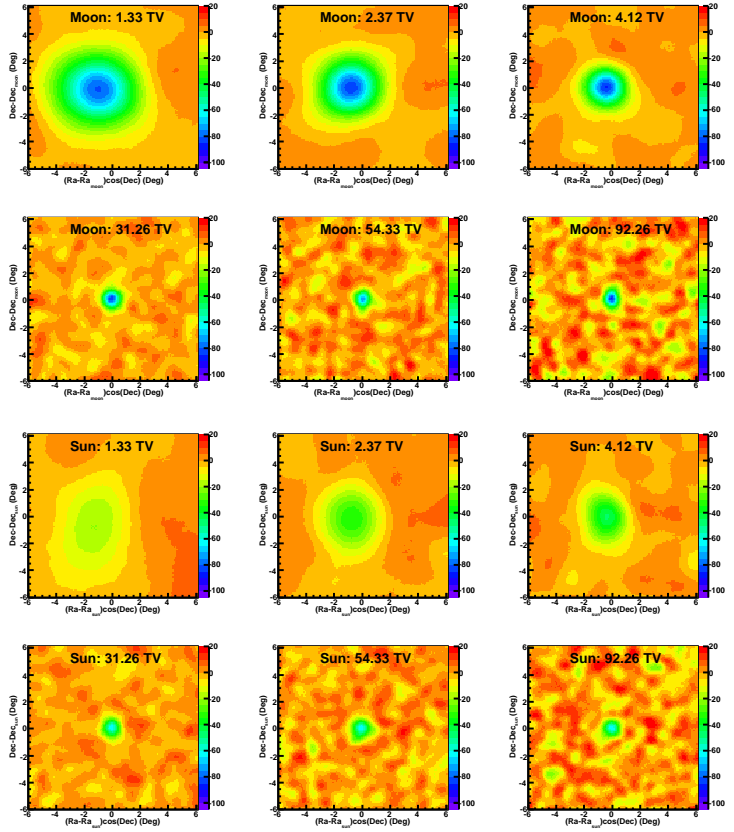
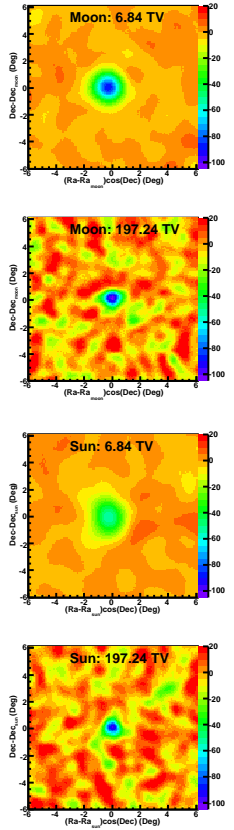

Figure 3: The deficit ratio (in percentage) map of the Moon and Sun shadow in the 12 energy bins using the five years data from 2008 to 2012 .

Figure 3 shows the deficit ratio map of $12^{\circ} \times 12^{\circ}$ region centered on the Moon and Sun nominal positions, in the 12 energy bins, using all the five years data collected from 2008 to 2012. Each pixel contains the ratio $S / S_{\text {ideal }}$, where $S$ is obtained by the minimization procedure previously described but with fixing the shadow position at this pixel, $S_{\text {ideal }}$ is evaluated by the number of background events. Both the Moon shadow and Sun shadow are clearly detected in all energy 
bins. In low energy bins, the deficit ratio of Sun shadow is lower than that of Moon shadow. The difference decreases with increasing energy.

The Moon shadow is mainly affected by the GMF, which is stable. To check the quality of yearly data, the deficit ratio and displacement of Moon shadow are investigated. The results are reported in Figure 4, that shows that ARGO-YBJ data are stable in the 5 year period considered here. From the left panel of Fig 4, reporting the deficit ratio as a function of the rigidity, one can see that the ratio for rigidities below $1 \mathrm{TV}$ is lower than that at larger rigidities. This is mainly caused by the wider distribution of deflected angles in these bins, that causes a certain loss of deficit events, since the number of deficit events is estimated within a limited region. The loss of low energy deficit events also makes the median rigidities of deficit events in low energy bins to be slightly larger than those expected by simulations. The median rigidities of deficit events are reported in Table 1, where they can be compared with simulations. The right panel of Fig.4 shows the Moon shadow displacement as a function of the rigidity. In this figure the median rigidities are corrected to take into account the effect previously described. After this correction, the displacement $d$ follows the function $d=1.58^{\circ}$ (Rigidity/TV).
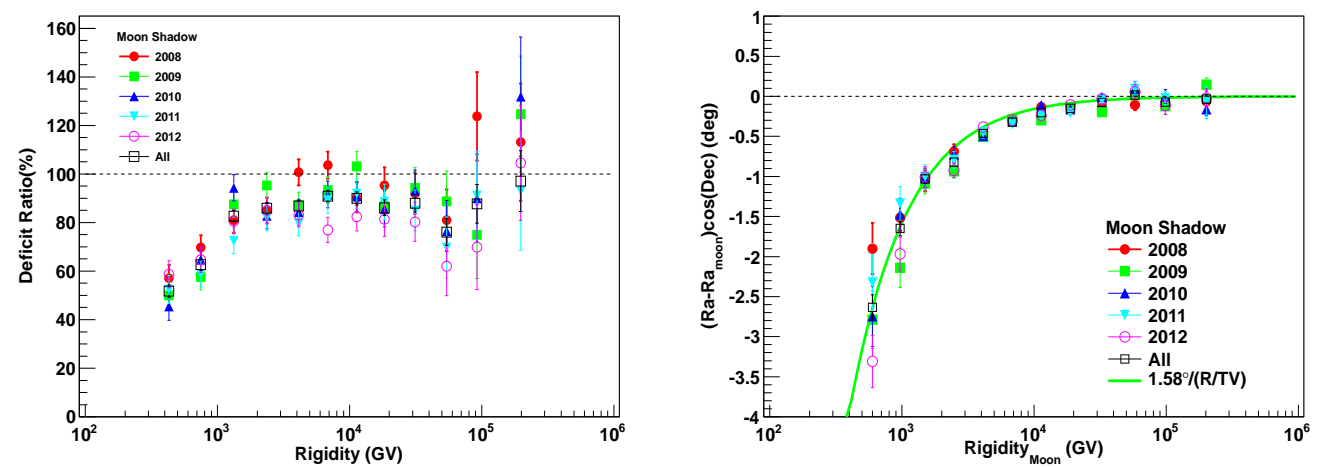

Figure 4: Left: The yearly deficit ratio of Moon shadow as a function of the median rigidity. Right: The yearly displacement of Moon shadow in the east-west direction as a function of the median rigidity.

Figure 5 shows the yearly deficit ratio of Sun shadow. During the period from 2008 to 2012, the solar activity varied from the minimum to the maximum. The deficit ratio is also gradually reduced with the enhancing of the solar activity. This variation is rigidity dependent. The influence is gradually reduced with increasing rigidity. To remove the effect of GMF and some possible data analysis technique, the deficit ratio of Sun shadow is normalized to the corresponding deficit ratio of Moon shadow, which is presented in the right panel of Figure 5. The variation of the normalized deficit ratio should be mainly caused by the SMF and IMF. During the minimum period of solar activity, the effect of SMF and IMF are remarkable only for events with rigidity lower than 2 TV. During the maximum period of solar activity, the effect can extend to a rigidity about $100 \mathrm{TV}$.

To quantitatively describe the deficit ratio as a function of the rigidity, we construct a sigmoid function to fit the deficit ratio as shown in the right panel of Figure 5. That is:

$$
y=\frac{100}{1+e^{-C *\left(\log (x)-\log \left(R_{50}\right)\right)}}
$$



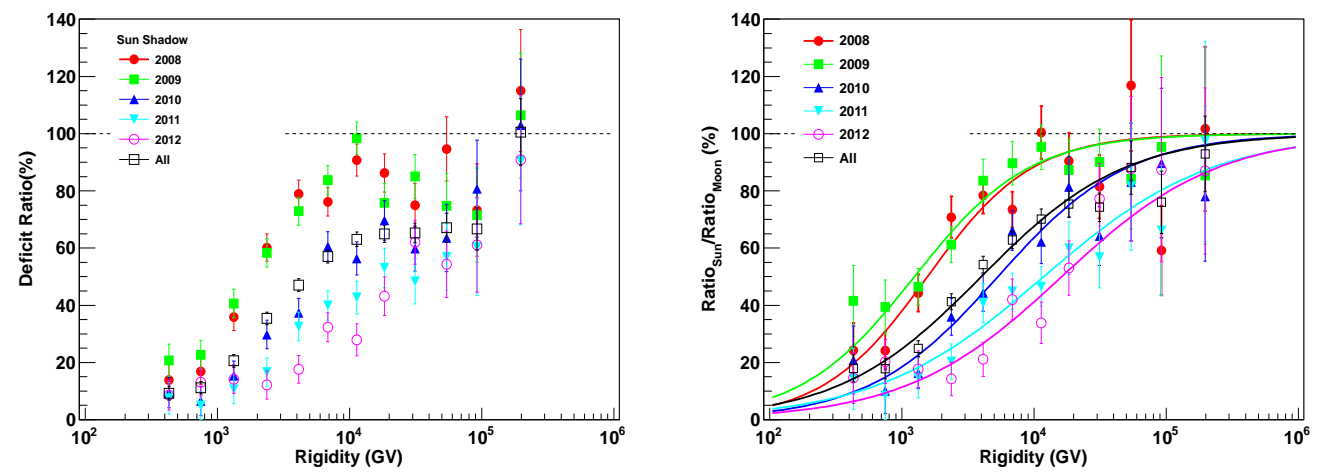

Figure 5: Left: The yearly deficit ratio of Sun shadow as a function of the median rigidity. Right: The normalized deficit ratio of Sun shadow.

where $\mathrm{C}$ describes the curvature of the sigmoid function and $\mathrm{R}_{50}$ is a characteristic rigidity with deficit ratio of 50\%. As shown in Figure 5, this function fits the data well. During 2008 and 2009 , the characteristic rigidity $R_{50}$ are $1.6 \pm 0.2 \mathrm{TV}$ and $1.2 \pm 0.2 \mathrm{TV}$, respectively. The value of $\mathrm{R}_{50}$ increases to $11.8_{-2.3}^{+2.8} \mathrm{TV}$ and $16.2_{-3.5}^{+4.4} \mathrm{TV}$ in 2011 and 2012, respectively. Figure 6 shows the monthly and yearly sunspot number since 1996, which traces the solar activity. The relation between the characteristic rigidity and the sunspot number is also shown in the right panel of Figure 6. The relation follows a linear function, i.e., $\mathrm{y}=(0.168 \pm 0.022) \mathrm{x}+(0.71 \pm 0.19)$.
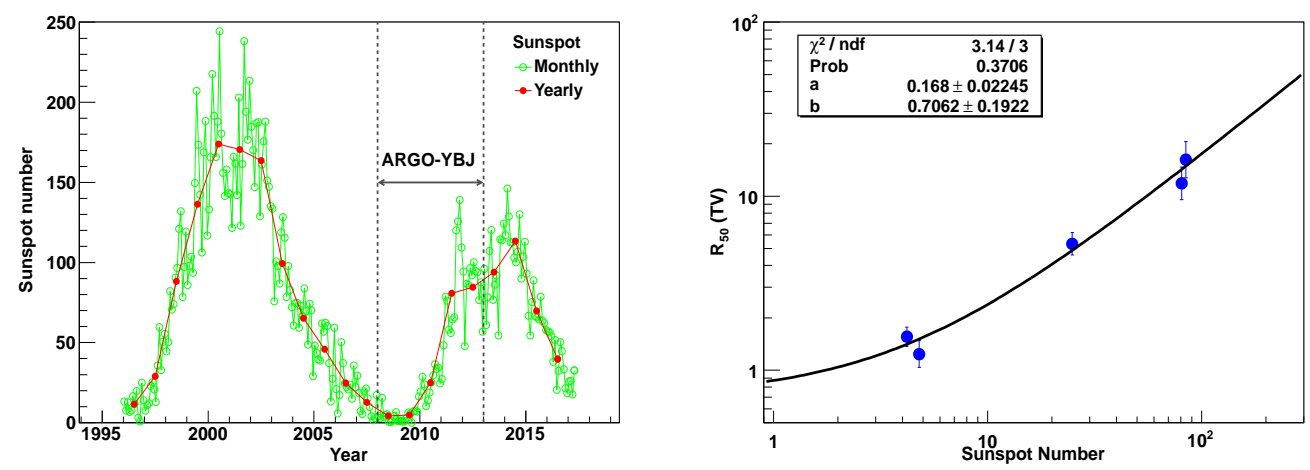

Figure 6: Left: The monthly and yearly sunspot number. Right: The characteristic rigidity vs the sunspot number.

\section{Discussion and Summary}

The correlation of deficit ratio with the solar activity has been confirmed by previous Tibet AS $\gamma$ []] and ARGO-YBJ [G] measurements at $10 \mathrm{TeV}$ and $5 \mathrm{TeV}$, respectively. In this work, we extend our study to the rigidity range from $0.42 \mathrm{TV}$ to $200 \mathrm{TV}$. The variation of Sun shadow is clearly observed. The Sun shadow is significantly reduced at rigidity below 2 TV even in the solar quiet phase. The relation between deficit ratio and rigidity generally follows a sigmoid function. The characteristic rigidity ranges from 1 TV to 16 TV during the period from 2008 to 2012 . The 
characteristic rigidity is linearly correlated with the sunspot number. With the obtained function, we can predict the Sun shadow basing on the sunspot number. It is worth to note that the solar activity in 2012 is much weaker than that in the year 2000 as shown in Figure 6. Basing on the data presented in Figure 6, the characteristic rigidity can be estimated to be around 30 TV during 2000. This is consistent with the Tibet AS $\gamma$ measurement showing that the Sun shadow almost disappeared in 2000 at $10 \mathrm{TeV}$.

With removing the effect of GMF, the variation of deficit ratio of Sun shadow should be mainly caused by the SMF and IMF. When cosmic rays reach the vicinity of Sun, the SMF could blow them out. This will cause the reduction of deficit shadow events. The IMF is generally parallel the ecliptic plane, hence it will deflect cosmic rays in the south-north direction. Because of the solar rotation, in a period of 27 days the IMF at the Earth position reverses (once or more times) its direction. This will cause a larger spread in the distribution of shadow events, which will reduce the deficit events within the fiducial region. According to our simulation, the IMF mainly affect the low energy bins, as shown in Figure 4. Therefore, the variation of deficit ratio presented in Figure 5 is dominated by the effect of SMF. In the future, we will quantitatively estimate the effect of IMF, to derive the pure effect of SMF. With these result, we can test the models of SMF year by year.

In summary, thanks to the high quality ARGO-YBJ data, the yearly rigidity dependent deficit ratio of Sun shadow has been measured for the first time. According to our data, the behavior of Sun shadow variation could be dominated by the solar magnetic field near the Sun.

\section{References}

[1] Clark,G. W. 1957, Phys. Rev. 108, 450

[2] Alexandreas, D. E., et al. 1991, Phys. Rev. D, 43, 1735

[3] Bartoli,B., Bernardini,P., Bi,X.J.,et al. 2011, Phys. Rev. D, 84, 022003

[4] Aielli, G., Bacci, C., B. Bartoli, B., et al. 2011, ApJ, 729, 113

[5] Amenomori, M., Bi, X. J., Chen, D., et al. 2013, PhRvL, 111, 011101

[6] Zhu, F.R., For the ARGO-YBJ Collab. 2011, 32nd International Cosmic Ray Conference

[7] Aielli, G., Assiro, R., Bacci, C., et al. 2006, Nucl. Instrum. Methods Phys. Res. A., 562, 92

[8] Heck, D., Knapp, J., Capdevielle, J. N., Schatz, G., \& Thouw, T. 1998, Forschungszentrum Karlsruhe Report FZKA, 6019

[9] Guo, Y. Q., Zhang, X. Y., Zhang, J. L., et al. 2010, ChPhC, 34, 555

[10] Gaisser T.K.,2012, Astroparticle Physics, 35, 801

[11] Aielli, G., Bacci, C., B. Bartoli, B., et al. 2009, APh, 30, 287

[12] Fleysher,R., Fleysher,L., Nemethy, P., \& Mincer, A. I. 2004, ApJ, 603, 355

[13] Li, T. P., \& Ma, Y. Q. 1983, ApJ, 272, 317

[14] James,F., and Roos,M. 1975, Computer Physics Communications, 10, 343 